\title{
A PROOF OF A CONJECTURE OF ERDÖS
}

\author{
BY RICHARD B. CRITTENDEN ${ }^{1}$ AND C. L. VANDEN EYNDEN ${ }^{1}$
}

Communicated by Paul T. Bateman, May 1, 1969

In $1958 \mathrm{~S}$. K. Stein [5] conjectured that if no $x$ satisfied more than one of the congruences

$$
x \equiv a_{i}\left(\bmod b_{i}\right), \quad b_{1}<b_{2}<\cdots<b_{n},
$$

then there existed an $x, 1 \leqq x \leqq 2^{n}$, satisfying none of them. P. Erdös proved this with $n 2^{n}$ instead of $2^{n}$ [1] and proposed the stronger conjecture that any system of $n$ congruence classes not covering all integers omits a positive integer not exceeding $2^{n}[1],[2],[3]$. Later John Selfridge proved Stein's conjecture [4].

We have proved Erdös's conjecture, and sketch the proof in this note. It is proper to mention that at the meeting of the American Mathematical Society in New Orleans in January 1969, Selfridge, in the course of a ten minute talk on another subject, made an informal preliminary announcement that he had also proved Erdös' conjecture.

Let us suppose the conjecture is false and that $n$ is the smallest number for which it fails.

Claim 1 . There exists a set of $n$ congruences such that

(A) each of the integers $1,2, \cdots, 2^{n}$ satisfies at least one of the congruences but 0 does not,

(B) all the moduli are prime, and

(C) if $k$ of the congruences have modulus $p$, then $2^{k}<p$.

PROoF. By our hypothesis there exist congruences $x \equiv a_{i}\left(\bmod b_{i}\right)$, $1 \leqq i \leqq n$, such that if $T$ is the set of integers satisfying none of the congruences, then $x \notin T, 1 \leqq x \leqq 2^{n}$, yet $T \neq \varnothing . T$ contains negative integers; let $x_{0}$ be the greatest nonpositive element of $T$. Then the congruences $x \equiv a_{i}-x_{0}\left(\bmod b_{i}\right)$ satisfy (A).

Now we assume we have $n$ congruences satisfying. (A). Suppose $x \equiv a(\bmod b)$ is one. Since $(\mathrm{A})$ implies $b \nmid a$, there exists a prime $p$ such that $p^{\alpha} \mid b$ but $p^{\alpha}\left\{a\right.$. Suppose $b=p^{\alpha} q$. Then we could replace this congruence with $x \equiv a\left(\bmod p^{\alpha}\right)$ without losing (A). Moreover, if $\alpha>1$ and $p \nmid a$, our original congruence could be replaced with $x \equiv a(\bmod p)$, still without losing (A). Thus we may assume all our congruences are of the form $x \equiv a\left(\bmod p^{\alpha}\right)$ (for various primes $p$ ), where $\alpha>1$ implies $p \mid a$. This is a start toward (B).

We illustrate our proof of (C) by taking the case $p=2$. By the last paragraph we can assume our congruences are of three types:

${ }^{2}$ Supported in part by NSF grants GP 6663 and GP 8075. 
(1) $x \equiv a(\bmod b), b$ odd,

(2) $x \equiv 1(\bmod 2)$, and

(3) $x \equiv 2 a\left(\bmod 2^{\alpha}\right), \alpha>1$.

If the type (2) congruence occurs, then each of the $2^{n-1}$ even integers between 1 and $2^{n}$ must be a solution to at least one of the remaining $n-1$ congruences of types (1) and (3). The same can be said if we replace each type (1) congruence $x \equiv a(\bmod b)$ by $x \equiv a+\epsilon b(\bmod 2 b)$, where $\epsilon=0$ if $a$ is even and 1 if $a$ is odd, since no even solutions have been lost.

We now have $n-1$ congruences of the form $x \equiv 2 a(\bmod 2 b)$, having among their solutions $2,4,6, \cdots, 2^{n}$ but not 0 . Then each of $1,2, \cdots, 2^{n-1}$ is a solution to one of the $n-1$ congruences $x \equiv a$ $(\bmod b) ; 0$ still is not. This contradicts our assumption that $n$ is the least integer for which the conjecture fails. The proof of $(\mathrm{C})$ for arbitrary prime $p$ is analogous.

Now (B). If $p$ is a fixed prime we know we can assume our congruences are of types

(1) $x \equiv a(\bmod b), p \nmid b$,

(2) $x \equiv a(\bmod p), p \nmid a$, and

(3) $x \equiv a\left(\bmod p^{\alpha}\right), \alpha>1, p \mid a$.

Since $2^{p-1} \geqq p,(C)$ tells us there exists $x_{0}$ such that $p \nmid x_{0}$ and $x_{0}$ solves no type-(2) congruence. Let $M$ be the product of the moduli of the type-(1) congruences and choose $r$ such that $r M \equiv x_{0}(\bmod p)$. It is easily checked that $r M$ satisfies no congruence, even if we change the modulus of each type (3) congruence from $p^{\alpha}$ to $p$, since $p \nmid r M$. This loses (A), but (A) can be regained by a shift just as at the beginning of this proof. Q.E.D.

Claim 2. Suppose $S_{1}, S_{2}, \cdots, S_{t}$ are sets of integers such that $S_{i}$ consists exactly of $k_{i}$ residue classes modulo $b_{i}, 1 \leqq i \leqq t$, and that $\left(b_{i}, b_{j}\right)=1$ if $i \neq j$. Then if $1 \leqq s \leqq t$ and $N$ is the number of integers $x$, $1 \leqq x \leqq 2^{n}$, such that $x$ is in none of the $S^{\prime}$ s, we have

$N>1+2^{n}\left(1-\sum_{i=0+1}^{t} k_{i} / b_{i}\right) \prod_{i=1}^{s}\left(1-k_{i} / b_{i}\right)$

$$
-\left(1+\sum_{i=+1}^{t} k_{i}\right) \prod_{i=1}^{i}\left(1+k_{i}\right) .
$$

Proof. Let $N_{i j} \ldots$, be the number of integers $x, 1 \leqq x \leqq 2^{n}$, in $S_{i} \cap S_{j} \cap \cdots \cap S_{z}$. It is well known that

$$
N=2^{n}-\sum_{i=1}^{t} N_{i}+\sum_{i} N_{i j}-\sum_{i, j, k} N_{i j k}+\cdots \cdot
$$


If $C\left(S_{i}\right)$ is the characteristic function of $S_{i}$, it is easily seen that $\left(1-\sum_{i=s+1}^{i} C\left(S_{i}\right)\right) \leqq \prod_{i=s+1}^{i}\left(1-C\left(S_{i}\right)\right)$. From this and

$$
\sum_{r=1}^{2 n} C\left(S_{i} \cap S_{j} \cap \cdots \cap S_{z}\right)(r)=N_{i j} \ldots s
$$

we conclude that the right side of (1) is $\geqq$

$$
2^{n}-\sum_{i=1}^{t} N_{i}+\sum_{i, j}^{\prime} N_{i j}-\sum_{i, j, k}^{\prime} N_{i j k}+\cdots,
$$

where $\sum^{\prime}$ means that only terms with at most one subscript $>s$ are added in.

Since the $b$ 's are relatively prime in pairs the Chinese remainder theorem implies that $N_{i j} \ldots z$ counts the solutions of $k_{i} k_{j} \cdots k_{z}$ congruences modulo $b_{i} b_{j} \cdots b_{z}$. Thus $N_{i j} \cdots z=2^{n} k_{i} k_{j} \cdots k_{z} / b_{i} b_{j} \cdots b_{z}$ $+E_{i j} \ldots z$, where $\left|E_{i j} \ldots z\right|<k_{i} k_{j} \ldots k_{z}$. Substituting this in (2) gives

$$
N \geqq 2^{n}\left(1-\sum_{i=1}^{t} k_{i} / b_{i}+\sum_{i, j}^{\prime} k_{i} k_{j} / b_{i} b_{j}-\cdots\right)+E,
$$

where $|E|<1+\sum_{i=1}^{t} k_{i}+\sum_{i, j}^{\prime} k_{i} k_{j}+\cdots$. The claim follows directly. Q.E.D.

The rest of the proof consists in showing that given a set of congruences as in Claim 1 we can apply Claim 2 so as to prove $N>0$, in contradiction to (A). We sketch the proof for $n \geqq 20$; smaller $n$ can be handled by special arguments. Let there be $k_{i}$ congruences modulo $p_{i}$ for $1 \leqq i \leqq t$, where the $p^{\prime}$ 's are prime and $p_{1}<p_{2}<\cdots<p_{t}$. We take $s=[n / 3]-1$. (If this is more than $t$ everything works with $s=t-1$.) We will show

$$
2^{n}\left(1-\sum_{i=s+1}^{t} k_{i} / p_{i}\right) \prod_{i=1}^{i}\left(1-k_{i} / p_{i}\right)
$$

$$
\geqq\left(1+\sum_{i=+1}^{i} k_{i}\right) \prod_{i=1}^{\dot{1}}\left(1+k_{i}\right) .
$$

The right side of (3) has $s+1$ factors with sum $n+s+1$ and so is maximized by $((n+s+1) /(s+1))^{s+1} \leqq 4^{n / 8}$. From inspection of a table of primes and known theorems we have $\pi(n-s) \leqq[n / 3]$ for $n \geqq 20$; from this we conclude $\sum_{i-s+1}^{t} k_{i} \leqq n-s<p_{[n / 3]}$. Then letting $p_{0}=p_{[n / 3]}$ and $k_{0}=\sum_{i=s+1}^{t} k_{i}$ we have the left side of $(3)$ is $\geqq 2^{n} \prod_{i=0}^{i}\left(1-k_{i} / p_{i}\right)$, where $k_{i} \leqq\left[\log _{2} p_{i}\right], 1 \leqq i \leqq s$. By elementary inequalities this product can be seen to exceed 


$$
2^{n}\left(1-\frac{1}{3}\right)\left(1-\frac{2}{5}\right)\left(1-\frac{2}{7}\right)\left(1-\frac{3}{11}\right) 13^{(8-n) / 12} .
$$

This is easily seen to be greater than $4^{n / 3}$ for $n \geqq 20$.

\section{REFERENCES}

1. P. Erdös, Extremal problems in number theory, Mat. Lapok 13 (1962), 228-255.

2. - Problems 29 and 30, Proc. No. Th. Conference, Boulder, Colorado, 1963, p. 96.

3. - Extremal problems in number theory, Proc. Sympos. Pure Math., vol 8 Amer. Math. Soc., Providence, R. I., 1965, p. 183.

4. J. Selfridge, On congruences covering consecutive integers, Acta Arith. (to appear). 5. S. K. Stein, Unions of arithmetic sequences, Math. Ann. 134 (1958), 289-294.

The Pennsylvania State University, University Park, Pennsylvania 16802 AND

Ohio University, Athens, Ohio 45701 